\title{
NONLINEAR INVERSION FOR THE HYDROSTATIC STRUCTURE OF THE SOLAR INTERIOR
}

\author{
K.I. MARCHENKOV, I.W. ROXBURGH AND S.V. VORONTSOV \\ Astronomy Unit, Queen Mary College, University of London \\ Mile End Road, London E1 4NS, UK
}

The technique which we use is based on the "quasi-asymptotic" description of solar p-modes developed by Roxburgh \& Vorontsov (1996). As a significant improvement of the standard asymptotic analysis, this description incorporates a Born approximation to allow for a detailed treatment of the regions of rapid variation of seismic parameters with depth (base of the convection zone) and strong influence of gravity perturbations (low-degree modes which penetrate deep into the solar core).

Essentially the eigenfrequency equation used is approximated by a second-order inhomogeneous Airy equation with a frequency dependent "acoustic potential", which is solved by a Born-type perturbation analysis. Matching the solutions in the interior with "exact", non-asymptotic solutions in the surface layers, where asymptotic analysis becomes locally invalid, leads to the eigenfrequency equation

$$
\int_{r_{1}}^{R} s d r \simeq \frac{\pi}{\omega}\left(n+\frac{5}{72} \frac{1}{\pi^{2} n}+\alpha^{\text {int }}+\alpha^{o u t}\right), \quad s^{2}=\frac{1}{c^{2}}-\frac{\tilde{w}^{2}}{r^{2}}, \quad \tilde{w}=\frac{\ell+1 / 2}{\omega} .
$$

The outer layers contribute to the eigenfrequency equation with a surface phase shift $\alpha^{\text {out }}$, similar to that of the standard asymptotic analysis. $\alpha^{\text {int }}$ is the frequency and degree dependent internal phase shift, given in the first order by an integral over the interior of the acoustic potential weighted with Airy function squared, $r_{1}$ denotes the position of the turning point $s^{2}\left(r_{1}\right)=0, c^{2}$ is squared sound speed, $\omega$ is frequency.

To develop an inversion technique based on this analysis we proceed as follows. First of all, we infer the leading-order approximation to $F(\tilde{w})=\int_{r_{1}}^{R} s d r$ by putting $\alpha^{i n t}=0$. In this stage we separate the input data $(\pi n / \omega)$ in the right-hand side of equation (1) into three parts: a) the leading-order asymptotic term $F(\tilde{w}), b)$ the frequency dependent surface phase shift for low-degree modes $\left.\alpha_{0}^{\text {out }}(\omega), \mathrm{c}\right)$ the firstorder correction to the surface phase shift for intermediate and high degree modes $\tilde{w}^{2} \alpha_{2}^{\text {out }}(\omega)$. Secondly, we invert $F(\tilde{w})$ using standard Abel integral transform to get first approximation for the sound-speed profile. After that we use the equations of hydrostatic support to determine the Brunt-Vaisala frequency profile and calculate the internal acoustic Born potential and corresponding Born phase shift for each mode used in the analysis. With the surface phase shift determined in the first step and with the internal phase shift determined in the previous step we infer an improved value of $F(\tilde{w})$ from equation (1) and an improved estimate of the sound speed-profile. 
We repeat the above steps until there is no change in the sound speed profile between successive iterations.

The accuracy, stability and convergence of the iteration scheme have been investigated in considerable detail using artificial data (frequencies) sets computed for solar models. The procedure is stable and converges in few iterations.

The numerical resuls are presented in the following figures.

a) : High resolution Inversion

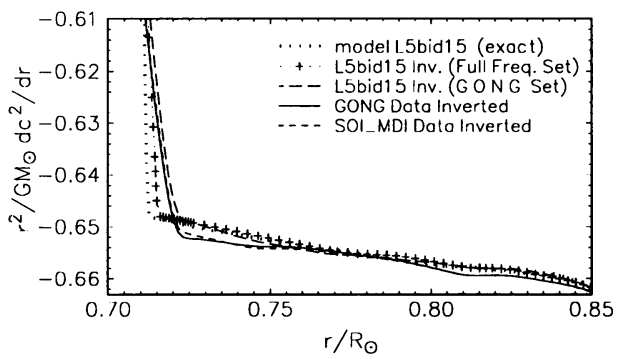

b): Global Sound Speed Inversion

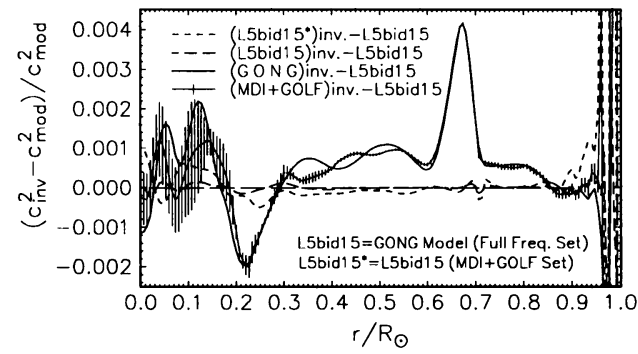

Figure 1. a) Sound speed gradient inversion. b) Global sound speed inversion. Vertical bars mark the SOHO curve and show the envelope of the solutions, when the white noise of the reported observational error amplitudes was added to the oscillation frequencies, in 100 random realizations.

The GONG data set used consists of 1803 modes with frequencies in the range $1 \leq \omega /(2 \pi) \leq 4.5 \mathrm{mHz}$. There may be further information in the data at higher and lower frequencies, but the quality of GONG data at those frequencies is not good enough. In Figure 1a we compare the results of the inversions for the sound speed gradient $d c^{2} / d r$ obtained with GONG data, SOI/MDI data (144 days data set, 1802 modes used), and GONG reference solar model (Model S, 15bi.d.15, ChristensenDalsgaard et al., 1996) with the exact values derived from the model. The primary target of our analysis is to achive the highest possible resolution of the region around the base of the solar convection zone, for searching possible signatures of penetrative convection, element diffusion and/or strong magnetic fields. Hopefully, it should allow the direct calibration of equation of state in the Sun, given high quality data of intermediate degree range.

Also the Born approximation technique successfully incorporates the effects of gravity perturbations in the solar core and the inversion procedure is now being used to diagnose the structure of the deep interior, given high quality data on modes with low $\ell$ values. In Figure 1b we present preliminary results for global sound speed inversion obtained with GONG data and combined data set of SOI/MDI (144 days) and low $\ell$ GOLF (110 modes used) frequencies. The difference in results in the deeper regions between GONG and SOHO is clearly seen. Unfortunately MDI and GOLF data sets contain almost no frequencies lower than $1.5 \mathrm{mHz}$ and higher than $4.0 \mathrm{mHz}$, which restricts the resolution of our inversion.

The iterative inversion technique described here is fully nonlinear, uses only oscillation frequencies as the input data and does not use any reference solar model.

\section{References}

Christensen-Dalsgaard, J., et al.,: 1996, Science 272, 1286.

Roxburgh, I.W., and Vorontsov, S.V.: 1996, Monthly Notices Roy. Astron. Soc. 278, 940. 